\title{
Semilleros de investigación: desarrollos y desafíos para la formación en pregrado
}

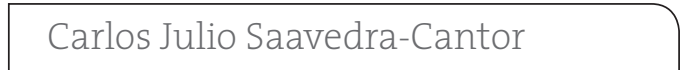

Universidad Nacional de Colombia (Colombia)

cajsaavedraca@unal.edu.co

\section{Carolina Antolínez-Figueroa}

Universidad Nacional de Colombia (Colombia)

cantolinezf@unal.edu.co

\section{Ana Helena Puerto-Guerrero}

Universidad Nacional de Colombia, (Colombia)

ahpuertog@unal.edu.co

\section{Alba Idaly Muñoz-Sánchez}

Universidad Nacional de Colombia (Colombia)

aimunozs@unal.edu.co

\section{Yurian Lida Rubiano-Mesa}

Universidad Nacional de Colombia (Colombia) ylrubianom@unal.edu.co

\section{Resumen}

Los semilleros de investigación se vienen consolidando como una estrategia extracurricular para la formación de investigadores desde el pregrado. El objetivo de esta investigación fue describir los desarrollos y desafios de los semilleros de investigación en la formación de estudiantes de pregrado en Colombia.

Se concluyó que los semilleros de investigación se caracterizan por ser escenarios alternativos de discusión académica e investigativa, fortalecen las habilidades investigativas de docentes y estudiantes, fomentan la interdisciplinariedad, favorecen el relevo generacional y orientan una proyección profesional en los estudiantes.

\section{Palabras clave}

Pedagogía Universitaria, formación de investigadores, enseñanza superior, instituciones de enseñanza, Educación superior-Colombia (Fuente: Tesauro de la Unesco).

Recepción: 2015-01-22 | Envío a pares: 2015-02-21 | Aceptación por pares: 2015-08-10 | Aprobación: 2015-08-15

DOI: 10.5294/edu.2015.18.3.2

Para citar este artículo / To reference this article / Para citar este artigo

Saavedra-Cantor, C. J., Muñoz-Sánchez, A. I., Antolínez-Figueroa, C., Rubiano-Mesa, Y. L., Puerto-Guerrero, A. H. (2015). Semilleros de investigación: desarrollos y desafíos para la formación en pregrado. Educ. Educ., 18 (3), 391-407. DOI: 10.5294/edu.2015.18.3.2 


\title{
Seedbeds of Research: Developments and Challenges in Undergraduate Training
}

\begin{abstract}
Seedbeds for research are being consolidated as an extracurricular strategy to train researchers beginning at the undergraduate level. The objective of this study was to describe the developments and challenges of seedbeds for research in undergraduate training in Colombia.

The conclusion is that seedbeds for research are characterized as alternative scenarios for academic debate and research, ones that strengthen the research skills of teachers and students, encourage interdisciplinarity, favor a generational shift, and orient the student's projection or potential as a professional.
\end{abstract}

\section{Keywords}

college teaching, training researchers, higher education, teaching institutions, higher education in Colombia (Source: Unesco Thesaurus). 


\section{Grupos de pesquisa: desenvolvimentos e desafios para a formação na graduação}

\section{Resumo}

Os grupos de pesquisa vêm se consolidando como uma estratégia extracurricular para a formação de pesquisadores a partir da graduação. O objetivo desta pesquisa foi descobrir os desenvolvimentos e desafios dos grupos de pesquisa na formação de estudantes de graduação na Colômbia.

Concluiu-se que os grupos de pesquisa caracterizam-se por serem cenários alternativos de discussão acadêmica e investigativa, fortalecem as habilidades de pesquisa de docentes e estudantes, fomentam a interdisciplinaridade, favorecem o relevo geracional e orienta uma projeção profissional nos estudantes.

\section{Palavras-chave}

pedagogia universitária, formação de pesquisadores, ensino superior, instituições de ensino, educação superior-Colômbia (Fonte: Tesauro da Unesco). 


\section{Introducción}

Los ejes primordiales de una universidad, como institución social, son la docencia, la extensión y la investigación, los cuales pretenden dar respuesta a las demandas de formación científica, tecnológica y humanística de las nuevas generaciones; al compromiso de construir soluciones a las necesidades de la población, y a la exigencia de aportar al desarrollo del conocimiento científico; estos tres ejes son complementarios e interdependientes, por lo que se deben desarrollar simultáneamente, para fortalecer los aportes de la universidad a la sociedad (Martínez, Mavárez, Rojas y Carvalho, 2008). La universidad es la llamada a construir los conocimientos sociales y a reforzar la relación de la academia con la sociedad, pero esto no se refleja en su totalidad, por la falta de trabajo interdisciplinario y por el modelo educativo, que prepara capital humano en distintas áreas para ser competitivo en el mercado laboral (Botero, 2009).

La Universidad de Humboldt (Alemania) se consolidó como un referente histórico en la formación investigativa de los jóvenes universitarios del mundo, debido a su modelo de cultivar las ciencias y las artes mediante la investigación; la influencia de este modelo alcanzó las universidades más prestigiosas de Colombia, en las cuales se ha promovido como estrategia la formación de semilleros de investigación, con el fin de generar una masa crítica que resuelva las necesidades nacionales de investigadores (Quintero, Munévar y Munévar, 2008).

La década de los setenta experimenta en Colombia una gran agitación política, que se gesta especialmente en las universidades públicas, donde los grupos extracurriculares de estudiantes, que trabajaban de manera cercana a los partidos y movimientos políticos de izquierda, se oponían enérgicamente a las políticas privatizadoras de la educación; esta época señala el comienzo de las comunidades de aprendizaje por fuera de los planes de estudio en las universidades. Varios de los temas que se discutían en estos escenarios políticos pasa- ron años después a debatirse en los semilleros de investigación (Tobasura, Jurado y Montes, 2009).

En Colombia, los planes curriculares previos a la década de los ochenta eran débiles en la formación investigativa, lo cual se expresaba en aspectos por mejorar por parte de los docentes y los estudiantes en el campo investigativo; por ello se impulsó la investigación y los proyectos comunitarios. En los años ochenta fue crucial el inicio de la formación investigativa; en los planes curriculares de pregrado se incluyeron tres componentes fundamentales: el investigativo, de formación socialhumanística y de formación profesional; de esta manera, se lograron fortalecer, en el ámbito universitario, los trabajos de grado y los seminarios, entre otros (Tobasura, et al., 2009).

Molineros señala que los orígenes de los semilleros de investigación se dan en la Universidad de Antioquia, en 1996, como fundamento de una estrategia extracurricular para el fomento de la investigación, y que posteriormente se propagaron por varias universidades del país. A nivel gubernamental, en el año 1998, el Departamento Administrativo de Ciencia Tecnología e Innovación (DACTI/Colciencias) auspició la creación de grupos académicos extracurriculares a través de su Política de Semilleros de Investigación. A inicios del nuevo milenio, Colciencias replanteó sus políticas, para consolidar la investigación científica, forjar conocimiento en los sectores estratégicos y fortalecer las capacidades científicas y tecnológicas nacionales. Simultáneamente, se incentivaron las redes de semilleros y la medición de grupos de investigación por Colciencias, y se solidificaron las alianzas y los eventos científicos, las comunidades virtuales de aprendizaje y la participación de estudiantes en investigaciones lideradas por docentes (Molineros, 2009).

En la actualidad, los semilleros de investigación se han convertido en una herramienta para proyectar desde el pregrado los futuros investigadores que necesita el país, con base en la convicción de que una 
nación se desarrolla y progresa a medida que lo hacen su ciencia, tecnología e innovación; con el avance de estas tres se consigue brindar alternativas a las distintas problemáticas que emerjan (Silva, Torres y Sarmiento, 2008).

De acuerdo con cifras del Observatorio Colombiano de Ciencia y Tecnología (OCyT), que es un referente de consulta para los actores del Sistema Nacional de Ciencia,Tecnología e Innovación (SNCTI), Colombia invirtió en actividades de ciencia, tecnología e innovación (ACTI), en el año 2002, el 0,3\% de su Producto Interno Bruto (PIB); en el 2010, el 0,46\%,y en el 2012, el 0,449\%. Por su parte, la inversión en Investigación y Desarrollo (I+D) en estos mismos años, fue del o,11, el 0,18 y el 0,17\% del PIB, respectivamente. En contraste, el promedio en América Latina y el Caribe para el año 2010 de inversión en ACTI fue del 1,09\% del PIB, siendo Brasil el país de la región que más invirtió en ACTI, con el 1,62\% de su PIB, cifra que es casi 4 veces mayor a la de Colombia (OCyT, 2012). Según el Banco Mundial (BM), la inversión en I+D en América Latina, en el 2010, fue del o,83\%,y el promedio mundial en el 2011 fue del $2,09 \%$. Al año 2010, el país latinoamericano que más invirtió en I+D fue Brasil, con el 1,16\% del PIB, seguido por Argentina, con el o,62\%, y Cuba, con el o,61\% (Banco Mundial, 2013).

El OCyT (2012) destaca que en el año 2002 se graduaron en Colombia 294 doctores; en el 2010, 629, y en el 2011, 644. En el año 2011, el número total de doctores en Colombia, en todas las áreas del conocimiento, era de 7129 (4914 hombres y 2215 mujeres). En el año 2002, el país contaba con 160 jóvenes investigadores en todas las áreas, y para el año 2011 Colombia contó con 1.000 nuevos jóvenes investigadores. En Colciencias se encuentran registrados 12.774 grupos de investigación, de los cuales, 8442 están avalados, y 5554 de estos se hallan reconocidos; de los grupos reconocidos, 4068 se encuentran categorizados: 216 en A1, 275 en A, 667 en B, 965 en C y 1945 en D. Como se observa, la formación de doctores y de nuevos jóvenes investigadores está creciendo; sin embargo, se está lejos de dotar al país y a sus instituciones de educación superior de la masa crítica que se requiere.

Una de las preocupaciones primordiales de Colciencias radica en establecer puentes de comunicación entre las universidades y el sector privado, puesto que la mayoría de los artículos científicos producidos en Colombia no tienen relación directa con el sector productivo, que ha sido debilitado con la liberalización de los mercados; se considera que al fortalecer la industria nacional se fomentará el desarrollo científico y tecnológico desde el sector privado (Organización para la Cooperación y el Desarrollo Económico, 2012). Cabe reconocer que la ciencia y la innovación no se desarrollan únicamente en las universidades, también lo hacen en los institutos públicos de investigación y en las empresas privadas; el consenso de los investigadores se centra en que la investigación universitaria ofrece muchas ventajas con respecto a centros de investigación aislados de la academia, especialmente cuando las universidades consiguen establecer lazos con el sector privado y los organismos públicos (Agencia Nacional para la Superación de la Pobreza Extrema, 2012). En este contexto, los semilleros de investigación se consolidan como una herramienta para formar los profesionales requeridos por las instituciones tanto públicas como privadas a fin de generar ciencia e innovación para el país.

Los avances de los semilleros de investigación en Colombia confluyen en la Red Colombiana de Semilleros de Investigación (RedCOLSI), organización pionera del desarrollo de procesos de investigación formativa a gran escala en Colombia. Desde 1998, cuando se realizó el primer Encuentro Nacional de Semilleros, en la ciudad de Manizales, la Red realiza anualmente, en el mes de octubre, este evento, en el cual se desarrollan políticas, planes y programas, y se verifica el nivel de conocimiento y arraigo de una cultura científica en el país (Fundación RedCOLSI, 2013). Simultáneamente, Colciencias creaba el programa de Jóvenes Investigadores e Innovadores, que abre las puertas al quehacer científico de los jóve- 
nes profesionales egresados de las universidades del país; jóvenes que deben mostrar talento, competencia e interés por la investigación y la innovación en diferentes áreas del conocimiento, para favorecer vínculos con centros de investigación que los incluyan en sus actividades y así apropiarse de los métodos y conocimientos científicos y tecnológicos (Colciencias, 2012).

Correa (2006), en un estudio que buscaba evaluar los semilleros de investigación alrededor de logros, carencias, dificultades y debilidades, mostró varios de los resultados, entre los cuales se destaca la participación constante de los estudiantes en eventos científicos a nivel local y nacional, espacios donde se socializan artículos científicos, ponencias y proyectos, entre otros; un gran avance en estos procesos es la participación institucional, ya que son las universidades las que facilitan los espacios a estudiantes y docentes para los encuentros de los semilleros de investigación. A pesar de estas fortalezas y avances en la promoción de la formación en investigación, Correa señala la permanencia de algunas dificultades que no permiten consolidar una cultura investigativa, asociadas a la gran deserción estudiantil, a la falta de compromiso de algunos participantes y al desinterés por descubrir e innovar, así como la ausencia de políticas investigativas que les garanticen viabilidad real, sostenibilidad y continuidad a estos procesos.

Con el objetivo de describir los desarrollos y desafíos de los semilleros de investigación en torno a la formación de estudiantes de pregrado en Colombia, se realizó la investigación que aquí se presenta.

\section{Metodología}

La investigación consistió en una revisión no sistemática de literatura; utilizó como fuentes bases de datos científicas, revistas científicas indexadas, libros electrónicos y páginas de organismos internacionales, gubernamentales y no gubernamentales, y de universidades públicas y privadas; de igual manera, utilizó documentos de bases de datos como la Biblioteca Virtual de la Salud, Academic Search Complet, Scielo y Redalyc. Se definió buscar documentos cuya fecha de publicación no superara los nueve años, puesto que el conocimiento encontrado alrededor de esta temática es escaso.

Conjuntamente, se utilizaron documentos de la Organización de las Naciones Unidas para la Educación, la Ciencia y la Cultura (Unesco) y de la Cátedra Manuel Ancízar "Educación Superior: debates y desafíos" de la Universidad Nacional de Colombia, datos del OCyT, del BM y del Ministerio de Educación de Colombia, entre otros.

\section{Contexto de la educación superior en América Latina y Colombia}

La globalización ha conllevado una de las épocas más interesantes e inciertas para la educación superior, así como desafíos y problemas al cuestionarse el ideal de lo público y del bien común; la globalización es un fenómeno irreversible y ha traído consigo el ánimo de lucro en las instituciones de educación superior (IES); no obstante, podría convertirse en un instrumento para construir una sociedad global que responda a ideales de solidaridad y de desarrollo humano y social (López, 2008).

La educación superior contribuye a un mayor bienestar, a la democracia e igualdad desde la ciencia, la cultura y el arte. Actualmente, en América Latina se destacan algunos cambios en cuanto a la educación, como las nuevas tecnologías, que se encargan de transformar los espacios de aprendizaje; el paso de las universidades públicas tradicionales de un solo campus urbano a varios en distintos lugares de sus naciones; el bajo aporte económico de los Estados para las universidades públicas en algunos países; de pocas escuelas privadas se ha pasado a un dominio de estas en muchos países, a partir de la concentración de capital humano y financiero de la empresa privada en el acceso a la educación superior; igualmente, de la escasa inves- 
tigación científica se ha pasado a múltiples institutos de ciencia. Estos cambios representan las tendencias de la educación superior en América Latina, en un escenario de enormes brechas sociales y económicas entre sus habitantes (Didriksson, Medina, Rojas, Bizzozero y Hemo, 2008). Cabe señalar que la mercantilización de la educación y los intereses de las empresas transnacionales han sumergido a la educación en un producto ligado directamente a las leyes del mercado, en contravía de la necesidad de que la educación y la investigación deben tener una orientación hacia la resolución de problemáticas sociales en beneficio de la mayoría de la población (Moreno y Ruiz, 2009).

Según cifras del Instituto Internacional para la Educación Superior en América Latina y el Caribe (IESALC), los países con mayor concentración de estudiantes en universidades públicas, entre el 75 y el 100\%, son: Cuba, Uruguay, Bolivia, Panamá, Honduras y Argentina; los países que tienen entre el 50 y el $75 \%$ de estudiantes en universidades privadas son Brasil, Chile, El Salvador, Colombia, Costa Rica, Nicaragua y República Dominicana; en una situación intermedia entre el sector público y privado se encuentran Ecuador, México, Venezuela, Paraguay, Perú y Guatemala. En cierta medida, existe en la región un aumento de la universidad privada; en contraste, el número de investigadores con el que cuenta la región se ubica principalmente en universidades públicas, con un $65,1 \%$; esto representa el 0,87\% de investigadores por cada mil habitantes de la población económicamente activa. Cabe señalar que las publicaciones científicas de América Latina representan tan solo el 2,6\% del total de publicaciones a nivel mundial (Gazzola y Didriksson, 2008).

A pesar de este panorama, García (2007) menciona que las denominadas macrouniversidades (públicas, nacionales y autónomas) fueron las que más crecieron en tamaño, en los procesos investigativosy en la generación de posgrados; por consiguiente, sobre las universidades públicas recayó el desarrollo de nuevas líneas de investigación, la vinculación con el aparato productivo y sus servicios y la formación de recursos humanos para la producción de nuevos conocimientos. Pese a que los sistemas de incentivos financieros para estimular la productividad de la investigación en la región, son pocos, se destacan la Coordinación de Perfeccionamiento de Personal de Nivel Superior (Capes) en Brasil, y el Fondo Nacional para la Ciencia y la Tecnología (Fonacit) en Venezuela. Brasil, fuera de los recursos públicos girados desde el Ministerio de Educación, cuenta con recursos propios de los Estados y municipios; además, las instituciones generan recursos propios y reciben apoyo de instituciones financieras de actividades científicas y tecnológicas (García, 2007).

Con respecto a las nuevas tecnologías educativas, el Centro Superior para la Enseñanza Virtual (CSEV), en un informe que pretendió definir las perspectivas tecnológicas de la educación superior en América Latina, del 2013 al 2018, coincide en que las aplicaciones móviles llegarán a ser de gran uso en los próximos tres años; el aprendizaje basado en juegos virtuales jugará un papel trascendental, y los cursos online masivos están convirtiéndose en una alternativa a los cursos universitarios tradicionales. Para potenciar al máximo estas herramientas virtuales se requiere una alfabetización de nuevos medios digitales a docentes y estudiantes en todas las disciplinas y profesiones, puesto que a menudo son los procesos pedagógicos tradicionales los que limitan un mayor uso de las nuevas tecnologías (Johnson, Adams, Gago, García y Martin, 2013).

En Colombia, según cifras del BM, en el año 2009 se invirtió el 4,7\% del PIB en educación; en el 2010, el 4,8\%, y en el 2011, el 4,4\%; Brasil, uno de los países de la región con los mejores indicadores en educación, invirtió el 5,6\% en el 2009, y el 5,8\%, en el 2010 (Banco Mundial, 2013). Por su parte, un documento del Banco de la República de Colombia, publicado en el año 2014, muestra que en las últimas dos décadas aumentó la cobertura en educación superior: la matrícula en el pregrado pasó de 487.448 estudiantes, en 1990, a 1.841.282 estudiantes en el 2012; 
la educación técnica y tecnológica pasó de 183.319 estudiantes, en 1990, a 622.745 en el 2012 (Melo, Ramos y Hernández, 2014). La tasa bruta de cobertura de la educación superior de América Latina para el año 2011 fue del 42,3\%, y la de Colombia fue del 40,3\%, cifra cercana al promedio latinoamericano; es importante destacar que la cobertura en Colombia se encuentra lejos de países como Cuba, con el $80 \%$, y Puerto Rico, con el $86 \%$ (Melo et al., 2014). La deserción en la educación superior es preocupante, su tasa en el nivel técnico es acumulativa y alcanza el 33,4\% en el primer semestre y el 71,1\% en el décimo; en el nivel universitario, la tasa de deserción asciende de 18,6\% en el primer semestre a 47,0\% en el décimo, acumulativamente; el Ministerio de Educación Nacional (MEN) encontró como razones a la deserción la situación laboral inestable y falta de recursos económicos (Melo et al., 2014).

Así mismo, el Banco de la República de Colombia destaca que durante la última década la financiación estatal para la educación superior no ha mostrado cambios significativos respecto a la inversión del PIB, que al año 2012 para educación superior fue del o,87\%, esto claramente denota que el Estado no ha respondido de forma simultánea al crecimiento de la demanda poblacional por cupos en la educación superior (Melo et al., 2014). En la clasificación mundial de instituciones de educación superior por número de publicaciones indexadas en Scopus durante el periodo 2007-2011, la Universidad Nacional de Colombia se ubica como la mejor del país, en el puesto 41, con 5.112 publicaciones, seguida por la Universidad de Antioquia, en el puesto 71, con 2.721 publicaciones, y la Universidad de los Andes, en el puesto 92, con 2.049 publicaciones (Melo et al., 2014).

En este contexto, los semilleros de investigación para estudiantes de pregrado se forman primordialmente en las universidades, que han experimentado una serie de transformaciones a partir de la globalización capitalista y presentan distintas crisis ante la disminución de la financiación de los Estados. De Sousa y Rivera (2006) plantean que ante el reto inaplazable de la privatización de la universidad pública, es necesario formular otras alternativas, como replantear nuevos contratos políticos y sociales en los cuales la universidad pública recupere su papel en la resolución colectiva de problemáticas sociales mediante la investigación, cuyos fines científicos y de producción de conocimiento están estrechamente ligados a la satisfacción de las necesidades de la sociedad.

\section{Conceptualización de los semilleros de investigación}

A pesar de que no existe un consenso general sobre el concepto de los semilleros de investigación, se presentan aquí algunas definiciones de autores e instituciones, con el objetivo de generar aproximaciones conceptuales.

Molina, Martínez, Marín y Vallejo (2012) subrayan la existencia de diferentes tipos de semilleros de investigación, que obedecen a las formas como desarrollan sus actividades en la investigación formativa; pero cualquiera que sea la metodología, se caracterizan por propiciar espacios agradables para sus participantes, de seguridad y solidaridad, en los cuales se generan preguntas orientadas al desarrollo de competencias en investigación; asimismo, son espacios donde confluyen el estudiante y el docente, encaminados a la iniciación de comunidades de aprendizaje a través de la formulación de objetivos, temas y metas, por lo cual asumen una responsabilidad social y pertenencia en la labor investigativa. García (2010) señala que un semillero de investigación garantiza el relevo generacional e institucional, facilita el fortalecimiento de la excelencia académica, la interdisciplinariedad y promueve el aprendizaje en el pregrado.

Gonzales (2008) enfatiza en que los semilleros de investigación son una estrategia para la formación integral de quienes los conforman, para crear una cultura investigativa en la comunidad; forjan herramientas investigativas y desarrollan habilida- 
des metodológicas, cognoscitivas y sociales; esta estrategia se basa en una constante interacción entre los docentes y estudiantes en un ambiente de diálogo e intercambio de saberes que beneficia a la comunidad académica. Hernández (2005) plantea que los semilleros de investigación son espacios extracurriculares de formación científica, son grupos autogestionados y autónomos, conformados por estudiantes con motivación para iniciar o fortalecer su formación en investigación; además, brindan una resignificación y revaloración de sus principios, valores y actitudes en pro de establecer una labor científica.

Torres (2005), por su parte, define los semilleros de investigación como grupos formados en su mayoría por alumnos decididos a trazar un nuevo modelo de enseñanza y aprendizaje; son un lugar donde se fomenta la libertad, la crítica académica, la creatividad y la innovación; de esta manera, un semillero no se encarga únicamente de generar conocimiento, es el espacio ideal para fomentar en sus integrantes el pensamiento. Herrera (2013) plantea que los semilleros de investigación se caracterizan por ser escenarios de formación integral orientados al estudiante que inicia su inmersión en la investigación, y que parten de preguntas y problemas formulados por estudiantes; en resumidas cuentas, se aprende a investigar investigando, afirma el autor.

Dado que dentro de las instituciones educativas de educación superior es donde, mayoritariamente, se crean y desarrollan semilleros de investigación, la Dirección de investigadores de Universidad Católica de Colombia define que son ambientes o escenarios diseñados para identificar y reafirmar la vocación investigativa en un área específica del conocimiento, y agrega que los semilleros de investigación son aquella fuente primaria para la conformación de comunidades investigativas y estarán en manos de su espiritu investigador y de su capacidad de comunicación para la existencia de tal espacio dinamizador de conocimiento. Para la Universidad de Cundinamarca (2013), un semillero de investigación se caracteriza por ser una comunidad de aprendizaje constituida por estudiantes y docentes vinculados a una institución de educación, o a un organismo de investigación público o privado; el grupo formaliza su existencia por medio de un acta de constitución y la construcción de un plan de desarrollo enmarcado en tres ejes: la formación en investigación, el quehacer investigativo de corte formativo aplicado y el trabajo en equipo.

En esta misma línea, para Aldana (2010), los semilleros de investigación son grupos líderes para formar espíritu investigativo, los cuales se utilizan como una estrategia de los docentes para la construcción y reconstrucción del conocimiento científico, con el fin de incentivar las capacidades investigativas de sus miembros. Londoño (2005) destaca que los semilleros de investigación se forman a partir de la suma de intereses investigativos de los miembros de una comunidad académica, y luego empiezan a realizar actividades grupales de autoformación y divulgación científica. De igual manera, los miembros empiezan a incursionar en el campo de la investigación mediante la orientación de un docente y, posteriormente, el grupo recoge los frutos del trabajo colectivo para brindar soluciones a problemáticas sociales.

Ríos (2009) define los semilleros de investigación como nuevos modelos de enseñanza-aprendizaje que generan conocimiento y cualifican intelectual y creativamente a los estudiantes, promoviendo el surgimiento de nuevas escuelas de pensamiento. A su vez, agrega el autor, los semilleros de investigación son una práctica de diálogo permanente, que discute temas de interés y de trascendencia para la salud y la vida, y con ellos los estudiantes inician en su carrera profesional un proyecto de vida ligado a la investigación.

\section{Importancia y aportes de los semilleros de investigación}

Los semilleros de investigación permiten un ambiente interdisciplinario, a partir de la interlocución entre facultades, saberes y culturas de los individuos 
que los conforman; pertenecer a estos grupos trae como beneficio un acceso mayor a servicios educativos, el uso adecuado de tecnologías existentes en la comunidad académica, así como la elección y focalización de una vida universitaria más comprometedora y productiva, ya que al iniciar tempranamente en el campo de la investigación se establece un puente entre el pregrado y el posgrado (Abello y Baeza, 2007). Los semilleros de investigación son la mejor estrategia para dar inicio a una vida ligada al conocimiento, para construir una cultura que prepare y genere hábitos investigativos en los estudiantes; en consecuencia, estos grupos buscan producir conocimiento para brindar identidad a la universidad o grupo de investigación al que pertenece el estudiante (Bolivar, 2013).

En concordancia, los semilleros de investigación generan un enriquecimiento académico y personal para las personas que voluntariamente decidieron incursionar en la vida investigativa; el académico es llevado a cabo cuando se cualifica el investigador con conocimiento y es capaz de realizar proyectos de investigación para resolver las problemáticas de su nación; por su parte, el personal se realiza cuando el investigador abstrae la realidad e interactúa con ella y su entorno (Cardona, Cano y Montes, 2007).

La Universidad Pontificia Bolivariana (UPB) (2012) menciona algunas finalidades de los semilleros de investigación: incentivar las competencias investigativas, impulsar una interrelación entre docentes, investigadores y estudiantes en pro de la excelencia académica y el desarrollo social. Paralelamente, los semilleros de investigación empoderan en temas científicos a sus integrantes y son espacios de formación extracurriculares, donde se desarrollan actividades como: grupos de estudio, grupos de discusión, construcción de textos, grupos de desarrollo, proyectos de investigación y divulgación de resultados científicos (Díaz, 2013).
A partir de su experiencia con el Semillero de Investigación de la Facultad de Medicina (Sifam), de la UPB, Martínez señala que los semilleros son indispensables para cumplir con el componente investigativo que hace parte de la formación profesional de los estudiantes; la experiencia de pertenecer al Sifam, anota el autor, ha generado resultados positivos para sus estudiantes y docentes, pues han sido reconocidos a nivel nacional por el desarrollo de trabajos científicos y han contribuido al desarrollo de un currículo disciplinario y académico en el campo investigativo (Martínez, 2008).

Para Gonzales (2006), los semilleros de investigación, al ser comunidades de aprendizaje, deben brindar un espacio propicio para el diálogo y la expresión de pensamientos y sentimientos de sus integrantes, facilitando así el crecimiento personal y colectivo garantizado por las buenas relaciones interpersonales y el intercambio de conocimientos. De manera similar, los semilleros de investigación han sido esenciales para fabricar conocimiento y fomentar el trabajo en equipo desde la interdisciplinariedad, y tienen como resultado la formación de ciudadanos comprometidos con la sociedad.

Ossa (2009) destaca que los semilleros de investigación no son solo importantes para acceder a maestrías y doctorados, pues deben ser un motor para motivar, entusiasmar y fomentar el pensamiento crítico, puesto que se encargan de conservar despierta la curiosidad y el entusiasmo para investigar; estos espacios, agrega, retoman principios como el diálogo y la responsabilidad compartida en la formación, entregan a los estudiantes elementos para su desempeño como líderes sociales, manejan un enfoque multidisciplinar y alcanzan a traspasar las barreras institucionales; a su vez, desarrollan procesos de aprendizaje más por construcción, que memorísticos; promueven habilidades orales y escritas, y forman profesionales capaces de abordar problemas de manera crítica y con una visión social y democrática. 


\section{Desafios de los semilleros de investigación}

Las recientes transformaciones económicas, sociales, tecnológicas y científicas han determinado un reto para la formación educativa e investigativa de estudiantes en todas las áreas del conocimiento a escala mundial, el cual ha traído consigo la reformulación de planes de estudio en distintas universidades, a partir de una necesidad imperiosa de desarrollo tecnológico y científico para las sociedades actuales (Núñez, 2014).

Ante esta realidad contemporánea, se ha valorado que los jóvenes ya cuentan con conocimientos previos y que son los actores principales de su formación investigativa; a pesar de esto, aún perduran prácticas educativas unidireccionales de los docentes, las cuales retardan el aprendizaje; los semilleros de investigación pueden aportar al cambio de estas prácticas al propiciar nuevos modelos pedagógicos de interacción de conocimientos entre estudiantes y docentes (Patiño, 2007). Incluso, los avances tecnológicos, la comunicación constante de los jóvenes con sus pares y la información obtenida fácilmente en la red componen un reto para los docentes en la formación investigativa de los estudiantes, lugar en el cual los docentes deben procurar actuar como facilitadores del conocimiento y potenciar la capacidad de asombro juvenil en el tema investigativo (Escobar, 2013).

Rojas (2008) señala que el sistema educativo colombiano muestra en todos sus niveles baja calidad en ciencia, tecnología e innovación, porque el problema de la formación investigativa en niños, niñas y jóvenes no es solamente pedagógico; por el contrario, sus raíces se dan en un déficit de políticas públicas que fomenten la investigación a temprana edad en las escuelas y universidades. En concordancia, las políticas públicas deben buscar el tratamiento de problemas y la satisfacción de demandas sociales a través de las autoridades públicas con la incorporación, programación y ejecución de acciones para promover la investigación desde la niñez. Con respecto a este panorama, Malo (2007) diseñó e implementó un programa de inducción a la investigación que inicia en la escuela, con el fin de promover el desarrollo de las competencias necesarias previas a que el estudiante ingrese a la educación universitaria en pro de fortalecer las habilidades investigativas de niños, niñas y jóvenes.

La educación como institución social moderna, debe asumir el reto de comprender el contexto y la coyuntura social actual para enfrentar los crecientes problemas del campo social, entre los que sobresalen el impacto generado por la globalización en los sectores más vulnerables, la destrucción de la naturaleza, los peligros del cambio climático y la inequidad mundial en la distribución de los ingresos. Así, la educación, además de contribuir al desarrollo de la ciencia, la tecnología y la innovación, se encarga de promover un pensamiento crítico, reflexivo, creativo y socialmente responsable; esto es clave para reducir la pobreza y es una ruta para alcanzar los objetivos de las sociedades democráticas; sin embargo, para poder realizar lo mencionado, es indispensable que los Estados retomen su financiación (Salas, 2006).

Es importante mencionar que las sociedades actuales cuentan con un gran número de herramientas tecnológicas; la virtualidad y el buen uso de la información se convierten en elementos cruciales en la formación de investigadores; así pues, la virtualidad garantiza la existencia de los semilleros de investigación y facilita la comunicación cuando esta no pueda ser presencial, siempre y cuando se le dé un uso adecuado (Corpas, 2010).

Trujillo (2007), en un estudio realizado en la Universidad Católica de Colombia, definió cuatro desafíos para los semilleros de investigación en el país. El primero, se enfoca en la revolución tecnológica, partiendo de la accesibilidad de la población, sin importar su condición social, a las redes virtuales; se destaca que el no acceso a estas expresa desigualdad social. El segundo, se orienta a que la población conozca sobre sus derechos a obtener y crear conocimiento. 
El tercero, se refiere a crear conocimiento e innovación de carácter público, y no para generar propiedad privada. El último desafío es la recuperación de los saberes culturales propios de cada región, con el fin de crear conocimiento y resolver necesidades que satisfagan la problemática local, y no global, puesto que cada lugar está inmerso en un contexto diferente y la globalización contribuye a la generalización de las culturas, desvirtuando las prácticas y creencias propias de cada lugar.

Por su parte, Lozano, Ochoa y Restrepo (2012), en un estudio realizado por la Universidad de Antioquia, concluyeron que la investigación señala el camino para el desarrollo de la docencia y la extensión, y que es necesario contar con directrices institucionales encaminadas a la articulación de las funciones universitarias. De acuerdo con los hallazgos de este estudio, se puntualizan algunos desafíos, como por ejemplo: mantener y fortalecer los avances en la articulación de la docencia, la extensión y la investigación; definir una política de semilleros estudiantiles que conciba la articulación de estos con el plan de estudios, garantizando de esta manera su permanencia y desarrollo; optimizar el uso de las tecnologías de información y comunicación, y sistematizar experiencias exitosas de la articulación de la docencia, la extensión y la investigación en conjunto con los semilleros de investigación.

\section{Conclusiones}

De acuerdo con los hallazgos de este estudio, se puntualizan algunos desafíos, como por ejemplo: mantener y fortalecer los avances en la articulación de la docencia, la extensión y la investigación; definir una política de semilleros estudiantiles que conciba la articulación de estos con el plan de estudios, garantizando de esta manera su permanencia y desarrollo; optimizar el uso de las tecnologías de la información y la comunicación, y sistematizar experiencias exitosas de la articulación de la docencia, la extensión y la investigación en conjunto con los semilleros de investigación.
Ante las necesidades mundiales de desarrollo científico y tecnológico, los semilleros de investigación se consolidan como una herramienta capaz de generar los cimientos investigativos para los futuros profesionales que necesitan las naciones, solventando así múltiples necesidades en distintos sectores. Asimismo, los semilleros de investigación se han convertido en espacios en los que el estudiante pasa del modelo tradicional académico vertical a un modelo de aprendizaje que nace en la discusión, la iniciativa propia y el aprender a investigar investigando, siempre de forma conjunta con la orientación de un docente.

Los semilleros de investigación favorecen el relevo generacional de líneas de investigación y orientan una proyección profesional en los estudiantes. De igual manera, se caracterizan por ser escenarios alternativos de discusión académica e investigativa, fomentar la interdisciplinariedad y fortalecer las habilidades investigativas de estudiantes y docentes. En ellos convergen distintas posturas filosóficas y formas de abordar los fenómenos, las cuales facilitan encontrar soluciones a las problemáticas desde la diversidad.

Del mismo modo, la comunicación constante de los jóvenes con sus pares por medios electrónicos, y la información fácilmente obtenida en la red, conforman un desafío en la formación investigativa por parte de los docentes sobre los estudiantes, escenario sobre el cual se hace prioritaria una propuesta de cambio en la educación que contenga como uno de sus pilares centrales a los semilleros de investigación para propiciar el desarrollo científico y profesional.

Colombia cuenta con Colciencias, el organismo rector del SNCTI y encargado de medir la producción científica del país; a su vez, cuenta con la RedCOLSI, que procura estimular el desarrollo de la producción científica desde el pregrado; a pesar de estos desarrollos institucionales, en el país no figuran datos precisos sobre cuántos semilleros de investigación existen y cuántos pertenecen a grupos de investigación. 
Finalmente, ante los escasos artículos científicos encontrados sobre los semilleros de investigación, es imprescindible seguir avanzando en la producción científica sobre este tema, con el fin de compartir las experiencias de esta estrategia, conocer sus necesidades y tendencias, fortalecer el aprendizaje y propiciar una cultura investigativa que logre resolver las necesidades de la sociedad colombiana, sustentada en políticas nacionales e institucionales.

\section{Referencias}

Abello, R., y Baeza, Y. (2007). Estrategia de formación investigativa en jóvenes universitarios: caso Universidad del Norte. Revista STUDIOSITAS, 2 (2), 5-12.

Agencia Nacional para la Superación de la Pobreza Extrema (Anspe), Centro de Innovación Social (CIS) (2012). Estrategia Nacional de Innovación Social para Superación de Pobreza Extrema. Recuperado el 24 de agosto de 2014 de: http://www.politicadeinnovacionsocial.co/documents/250640/o/Estrategia+Nacional+de+l nnovacion+Social+baja-V2.pdf/8o8df995-668c-424e-8eb6-dfba3f4fec72

Aldana, L. (2010). Creando semilleros de investigación en la escuela. Revista Gondola [en línea], 5 (1), 3-10. Recuperado el 14 de agosto de 2014 de: http://comunidad.udistrital.edu.co/geaf/files/2012/og/2010Vol5No1-001.pdf

Banco Mundial (2013a). Gasto público en educación, total (\% del PIB). Recuperado el 26 de agosto de 2014 de: http://datos.bancomundial.org/indicador/SE.XPD.TOTL.GD.Z

Banco Mundial (2013b). Indicadores de ciencia y tecnología. Recuperado el 4 de septiembre de 2014 de: http:// datos.bancomundial.org/tema/ciencia-y-tecnologia

Bolívar, R. M. (2013). Los modos de existencia de la estrategia de semilleros en Colombia como expresiones de la comprensión de la relación entre investigación formativa y la investigación en sentido estricto. Múltiples lecturas, diversas prácticas. Revista El Ágora USB [en línea], 13 (2), 433-441. Recuperado el 4 de septiembre de 2014 de: http://web.b.ebscohost.com.ezproxy.unal.edu.co/ehost/pdfviewer/pdfviewer?sid=22986ab461fo-4621-a16e-23d5076d9587\%40sessionmgr111\&vid=o\&hid=114

Botero, S. A. (2009). Los semilleros de investigadores en la Universidad de Caldas. En Molineros, L. (ed.). Orígenes y dinámicas de los semilleros de investigación en Colombia: la visión de los fundadores (pp. 29-35). Colombia: Universidad del Cauca.

Cardona, M., Cano, C. y Montes, I. (2007). Formación en investigación en el pregrado: caso del semillero en Economía de la Universidad EAFIT. Revista STUDIOSITAS, 2 (3), 44-53.

Colciencias (2012). Bogotá DC: Colciencias. Recuperado el 10 de septiembre de 2014 de: http://www.colciencias. gov.co/faq/que-es-el-programa-de-jovenes-investigadores

Corpas, E. J. (2010). Virtualización de los semilleros de investigación: acaso un modelo de continuidad. Revista de Ciencia y Salud, 8 (2), 77-87. 
Correa, L. (2006). Estado actual del trabajo en red de los semilleros de investigación en Colombia. Revista opinión jurídica [en línea], 5 (10), 197-201. Recuperado el 14 de septiembre de 2014 de: http://www.scielo.org. co/scielo.php?script=sci_arttext\&pid=S1692-25302006000200011\&lang=pt

De Sousa Santos, B.y Rivera, R. (2006). La Universidad en el Siglo XXI. Para una reforma democrática y emancipadora. Revista Andamios, 3 (5), 307-311.

Díaz, L. M. (2013). Lineamientos para la construcción de semilleros de investigación. Secretaría Distrital de Salud. Recuperado el 14 de septiembre de 2014 de: http://www.saludcapital.gov.co/Documents/Investigaciones/Semilleros\%2ode\%2oinvestigaci\%C3\%B3n.pdf

Didriksson, A., Medina, E., Rojas, M., Bizzozero, L.y Hermo, J. (2008). Contexto global y regional de la educación superior en América Latina y el Caribe. Organización de las Naciones Unidas para la Educación, la Ciencia y la Cultura (Unesco). Instituto Internacional para la Educación Superior en América Latina y el Caribe-IESALC. Recuperado de: http://www.iesalc.unesco.org.ve/

Dirección de investigadores de Universidad Católica de Colombia. Procedimiento semilleros de Investigación POO4DICYTO1. Recuperado el 21 de septiembre de 2014 de: http://portalweb.ucatolica.edu.co/easyWeb2/ investigaciones/pdfs/programadesemillerosdeinvestigacion/ProSemInv.pdf

Escobar, M. V. (2013). Semilleros de Investigación. Revista de Investigación Andina, 15 (27).

Fundación RedCOLSI. (2013). Antioquia: Red Colombiana de Semilleros de Investigación. Recuperado el 21 de septiembre de 2014 de: http://www.fundacionredcolsi.org/index.php/institucional/resna-de-la-red

García, C. (2007). Financiamiento de la Educación Superior en América Latina. Revista Sociología de Porto Alegre, 9 (17), 50-101.

García, C. (2010). Uso de fuentes documentales históricas que favorecen la investigación formativa. El caso de los semilleros de investigación. Estudios Pedagógicos, 36 (1), 265-273.

Gazzola, A. L.y Didriksson, A. (2008). Tendencias de la Educación Superior en América Latina y el Caribe. Caracas: Organización de las Naciones Unidas para la Educación, la Ciencia y la Cultura (Unesco). Instituto Internacional para la Educación Superior en América Latina y el Caribe-lesalc.

Gonzales, J. (2008). Semilleros de investigación: una estrategia formativa. Revista Psychologia, Avances de la disciplina [en línea], 2 (2), 185-19o. Recuperado el 26 de septiembre de 2014 de: http://132.248.9.34/hevila/ Psy chologiaAvancesdeladisciplina/2008/vol2/no2/7.pdf

Gonzáles, Y. (2006). Los semilleros de investigación como comunidades de aprendizaje. Revista STUDIOSITAS [en línea], 1 (2), 34-36. Recuperado el 26 de septiembre de 2014 de: http://portalweb.ucatolica.edu.co/ easyWeb2/files/1_4o_los-semilleros.pdf

Hernández, U. (2005). Propuesta curricular para la consolidación de los Semilleros de Investigación como espacios de formación temprana en investigación. Revista Electrónica de la Red de Investigación Educativa [en línea], 1 (2), 1-12. Recuperado de: http://revista.iered.org/vin2/pdf/uhernandez.pdf 
Herrera, G.L.(2013).Tendencias actuales del proceso de formación de habilidades investigativas en estudiantes de la carrera de medicina. Revista de Ciencias Médicas [en línea], 17 (4),138-153. Recuperado el 28 de septiembre de 2014 de: http://scielo.sld.cu/scielo.php?script=sci_arttext\&pid=S1561-31942013000400015\&lang=es

Johnson, L., Adams, S., Gago, D., García, E.y Martin, S. (2013). NMC Perspectivas tecnológicas: educación superior en América Latina 2013-2018. Un análisis regional del Informe Horizon del NMC. Austin Texas: The New Media Consortium. Recuperado el 28 de septiembre de 2014 de: http://www.nmc.org/pdf/2013-technology-outlook-latin-america-ES.pdf

Londoño, A. F. (2005). Documento Conceptual de la Red Institucional de Semilleros de Investigación. Fundación Universitaria María Cano.

López, F. (2008). Tendencias de la educación superior en el mundo y América Latina y el Caribe. RevAvaliacao, Campina [en línea], 13 (2), 267-291. Recuperado el 28 de septiembre de 2014 de: http://www.scielo.br/pdf/ aval/v13n2/o3.pdf

Lozano, G. A., Ochoa, A. M.y Restrepo, S. L. (2012). La articulación entre investigación, docencia y extensión en un programa universitario de nutrición y dietética. Revista Perspectivas en Nutrición Humana [en línea], 14 (1), 71-83. Recuperado el 01 de octubre de 2014 de: http://www.scielo.org.co/scielo.php?script=sci_arttext\&pi $d=S 012441082012000100007$

Malo, D. A. (2007). Inducción a la investigación desde la educación básica como proyección a la educación superior. Revista STUDIOSITAS, 2 (3), 18-24.

Martínez, C., Mavárez, R., Rojas, L.y Carvalho, B. La responsabilidad social universitaria como estrategia de vinculación con el entorno social. Revista de Filosofía Jurídica, Social y Política, 15 (3), 81-103.

Martínez, L., Marvárez, R., Rojas, L.y Carvalho, B. Los semilleros de investigación, una estrategia para la formación en investigación. Revista Medicina UPB [en línea], 27 (1), 1-2. Recuperado el o1 de octubre de 2014 de: http:// www.redaly c.org/articulo.oa?id=159013072001)

Melo, L. A., Ramos, J. E.y Hernández, P. O. (2014). La educación superior en Colombia: situación actual y análisis de eficiencia. Banco de la República de Colombia, 1 (808), 1-50. Recuperado el o1 de octubre de 2014 de; http:// www.banrep.gov.co/docum/Lectura_finanzas/pdf/be_808.pdf

Molina, J. L., Martínez, L. M., Marín, A. E. y Vallejo, E. O. (2012). El semillero de investigación como una estrategia para la creación de aprendizaje autónomo en la Facultad de Medicina. Medicina UPB, 31 (2), 212-219.

Molineros, L. F. (2009). Epistemología de los Semilleros de Investigación y la Cultura en Red de la RedCOLSI: una visión compartida desde la experiencia de uno de sus actores. En Molineros, L. (ed.). Orígenes y dinámicas de los semilleros de investigación en Colombia: la visión de los fundadores (pp. 117-145). Colombia: Universidad del Cauca.

Moreno, J. C.y Ruiz, P. (2009). Comisión Económica para América Latina y el Caribe-Cepal. La Educación Superior y el Desarrollo Económico en América Latina. Serie de Estudios y Perspectivas. Recuperado el o5 de octubre de 2014 de: http://www.eclac.org/publicaciones/xml/5/35095/Serie_106.pdf 
ISSN 0123-1294 | Educ.Educ. Vol. 18. No. 3 | Septiembre-Diciembre de 2015 | pp. 391-407.

Universidad de La Sabana | Facultad de Educación

Núñez, J. (2014). La ciencia y la tecnología como proceso sociales. Recuperado el o5 de octubre de 2014 de: http://www.inder.cu/indernet/provincias/hlg/documetos/textos/P\%20S\%2ODE\%2OLA\%2OCIENCIA\%2O Y\%2LA\%2OTECNOLOGIA/P\%2OS\%2ODE\%2OLA\%2OCIENCIA\%2OY\%2OLA\%2OTECNOLOGIA.pdf

Observatorio Colombiano de Ciencia y Tecnología (2012). Indicadores de Ciencia y Tecnología, Colombia 2012. Recuperado el o5 de octubre de 2014 de: http://ocyt.org.co/eses/InformeAnuallndicadores/ArtMID/542/ ArticlelD/21/Indicadores-de-Ciencia-y-Tecnolog237a-Colombia-2012

Organización para la Cooperación y el Desarrollo Económico (OCDE). (2012). La educación superior en Colombia, evaluaciones de politicas nacionales de investigación. Recuperado el o7 de octubre de 2014 de: http://www. mineducacion.gov.co/cvn/1665/articles-317375_recurso_1.pdf

Ossa, J. (2009). ¿De dónde surgen la investigación? La “entusiasmina”y su contagiosidad. En Molineros, L. F. (ed.). Orígenes y dinámicas de los semilleros de investigación en Colombia: la visión de los fundadores (pp. 13-20). Colombia: Universidad del Cauca.

Patiño, J. F. (2007). Semillero Culturas Juveniles y Contemporaneidad: un escenario intersticial para la formación investigativa. Revista Cientifica Guillermo de Ockham, 5 (2), 137-140.

Quintero, J., Munévar, R. A. y Munévar, F. I. (2008). Semilleros de Investigación: una estrategia para la formación de investigadores. Revista de Educación y Educadores [en línea], 11 (1), 1-8. Recuperado el o7 de octubre de 2014 de: http://educacionyeducadores.unisabana.edu.co/index.php/eye/article/view/716/1694

Ríos, J. (2009). Hacia la formación de talento en investigación. Semilleros de investigación: una estrategia para abordar la ciencia. Revista Archivos de Medicina [en línea], 9 (1): 80-83. Recuperado el 10 de octubre de 2014 de: http://www.umanizales.edu.co/publicaciones/campos/medicina/archivos_medicina/html/publicaciones/edicion_9-1/11_semilleros.pdf

Rojas, H. M. (2008). La importancia de las políticas públicas de formación en investigación de niños, niñas y jóvenes en Colombia, para el desarrollo social. Revista Latinoamericana de Ciencias Sociales, Niñez y Juventud [en línea], 6 (2), 885-906. Recuperado el 10 de octubre de 2014 de: http://www.scielo.org.co/pdf/rlcs/ v6n2/v6n2a14.pdf

Salas, F. E. (2006). Las teorías de la modernidad reflexiva y de los sistemas sociales: aportes a la comprensión de las macrotendencias de la educación contemporánea. Revista Educación [en línea], 30 (2), 83-99. Recuperado el 10 de octubre de 2014 de: http://www.redaly c.org/articulo.oa?id=44030206

Silva, A. C., Torres, M. y Sarmiento, J. A. (2008). Dinámicas de los Semilleros de Investigación en la Universidad Militar de la Nueva Granada. Revista Facultad de Ciencias Económicas [en línea], 16 (1), 131-149. Recuperado el 10 de octubre de 2014 de: http://www.scielo.org.co/scielo.php?script=sci_arttext\&pid=S012168052008 ooo100010\&lang=es

Tobasura, I., Jurado, C. y Montes, C. M. (2009). Los grupos extracurriculares en agronomía de la Universidad de Caldas (Colombia): 1970-2006: de la efervescencia política a la formación científica. Revista Latinoamericana de Ciencias Sociales, Niñez y Juventud [en línea], 7 (2), 1573-1594. Recuperado el 10 de octubre de 2014 de: http://www.scielo.org.co/scielo.php?script=sci_arttext\&pid=S1692715X2009000300017 
Torres, L. C. (2005). Para qué los semilleros de investigación. Universidad Nacional de Colombia. Facultad de Ingeniería, 1-10. Recuperado el 15 de octubre de 2014 de: http://www.revistamemorias.com/edicionesAnteriores/8/semilleros.pdf

Trujillo, R. (2007). Los Semilleros de Investigación, una experiencia de formación en investigación para la inclusión social. Revista Educación en Ingeniería, 1 (3), 22-35.

Universidad de Cundinamarca (2013). Manual para la gestión de semilleros de investigación. Recuperado el 15 de octubre de 2014 de: http://intranet.unicundi.edu.co/investigacion/images/pdf/MINoo1_1\%2OManual\%20para\%2ola\%2ogestion\%2ode\%20semilleros\%2ode\%2olnvestigacion\%2o(version\%2ofinal).pdf

Universidad Pontificia Bolivariana (2012). Medellín: UPB. Recuperado el 15 de octubre de 2014 de: http://www. upb.edu.co/portal/page?_pageid=1054,32590779\&_dad=portal\&_schema=PORTAL 
